\title{
Static Tire/Road Stick-Slip Interactions: Analysis and Experiments
}

\author{
Yizhai Zhang, Student Member, IEEE, and Jingang Yi, Senior Member, IEEE
}

\begin{abstract}
Tire/road interactions play a critical role in safe operation of ground vehicles. This paper presents the modeling, analysis, and experimental studies of stick-slip interactions between the stationary tire and the firm road. A semi-analytical model is first proposed to predict the normal force distribution on the tire contact patch. A beam-spring network modeling approach is then used to capture and compute the friction force and rubber deformation distributions on the contact patch during a stick-to-slip transition. To validate the contact models and analyses, a pressuresensitive, electric conductive rubber sensor is embedded inside the tire rubber layer to extract the 3-D forces on the contact patch. Both the analytical and experimental results show that the friction force and rubber deformation distributions are dependent on the contact normal force distribution and the tire structural properties. The understanding and modeling of the static stick-slip interactions presented in this paper provide new knowledge and methods for studying dynamic tire/road interactions and flexible thin-layer/rigid contacts in other mechanical systems.
\end{abstract}

Index Terms-Contact model, force sensor, pneumatic tire, stickto-slip transition, tactile sensing.

\section{NOMENCLATURE}

$\mathcal{P}, \partial \mathcal{P}, \mathcal{P}^{\circ} \quad$ Tire/road contact patch, the boundary and the interior region of the contact patch.

$\mathbf{F}_{f} \quad$ Total tire/road friction force.

$F_{f x}, F_{f y} \quad$ Total friction forces along the $x$ - and $y$-axis directions.

$\mathbf{P}_{f} \quad$ Local friction stress on $\mathcal{P}$.

$P_{f x}, P_{f y} \quad$ Local friction stresses along the $x$ - and $y$-axis directions.

$F_{z} \quad$ Total tire/road normal contact force.

$P_{n} \quad$ Local normal contact pressure on $\mathcal{P}$.

$P_{\text {air }} \quad$ Tire inflation air pressure.

$\mathbf{f}_{f}^{i} \quad$ Friction force on beam $i$.

$\mathbf{f}_{b}^{i} \quad$ Bending force on beam $i$.

$\mathbf{f}_{e}^{i} \quad$ Net elastic force on beam $i$.

$\mathbf{F}_{\partial \mathcal{P}} \quad$ Total boundary force.

$F_{\partial \mathcal{P} x}, F_{\partial \mathcal{P} y}$ Total boundary forces along the $x$ - and $y$-axis directions.

Manuscript received February 1, 2013; revised May 15, 2013 and August 29 2013; accepted November 16, 2013. Date of publication December 17, 2013 date of current version June 13, 2014. Recommended by Technical Editor Y. Li. This work was supported in part by the U.S. National Science Foundation under Award CMMI-0856095. A preliminary version of this paper was presented in part at the 2012 IEEE/ASME International Conference on Advanced Intelligent Mechatronics, Kaohsiung, Taiwan, July 11-14, and the 2012 ASME Dynamic Systems and Control Conference, Ft. Lauderdale, FL, USA, October 17-19.

The authors are with the Department of Mechanical and Aerospace Engineering, Rutgers University, Piscataway, NJ 08854 USA (e-mail: yzzhang@ eden.rutgers.edu; jgyi@ rutgers.edu).

Color versions of one or more of the figures in this paper are available online at http://ieeexplore.ieee.org.

Digital Object Identifier 10.1109/TMECH.2013.2292872

\author{
$\mathbf{f}_{\partial \mathcal{P}}^{i} \quad$ Boundary force on beam $i$ on $\partial \mathcal{P}$. \\ $f_{\partial \mathcal{P} x}^{i}, f_{\partial \mathcal{P} y}^{i} \quad$ Boundary forces on beam $i$ on $\partial \mathcal{P}$ along the $x$ - \\ and $y$-axis directions. \\ $\sigma_{\mathcal{P} x}, \tau_{\mathcal{P} x} \quad$ Spatially normalized $\mathbf{f}_{e}^{i}$ and $\mathbf{f}_{b}^{i}$ along the $x$-axis \\ direction. \\ $2 h \quad$ Thickness of the tire rubber layer. \\ $R_{o}, R_{i} \quad$ Outer and inner radii of the tire rubber layer cross \\ the tire center line $\left(R_{o}=R_{i}+2 h\right)$. \\ $E, G, \nu \quad$ Young's modulus, shear modulus, and Poisson's \\ ratio of the tire rubber materials.
}

\section{INTRODUCTION}

$\mathbf{T}$ IRE/ROAD interactions play an extremely important role for vehicle safe operation. However, understanding and modeling of the tire/road interactions are challenging because these interactions are complex, highly nonlinear, and dependent on various tire and road conditions. Further challenges arise because of lack of effective, inexpensive sensing technology to obtain in-situ tire/road contact information [1].

Rubber deformations on the tire/road contact patch generate friction forces. Most empirical friction force models, such as Pacejka "magic" formula [2], capture the phenomenological relationships between total friction forces and tire kinematics (e.g., slip ratios, slip angles, etc.) Although the physical and the LuGre dynamic friction models [3]-[7] try to capture and interpret the total friction forces through rubber deformation distribution, several critical modeling assumptions are made $a$ prior. For example, the physical model in [3] uses an assumption of a simple division of adhesion (stick) and sliding (slip) regions of the tire contact patch. The LuGre dynamic models in [4]-[7] are built on the given normal force distribution to calculate rubber deformation distributions without experimental validation. Although the normal load distribution is discussed in [8] and [9], few works are reported on analyses and experiments of the rubber deformation and friction force distributions on the contact patch. One of main goals of this study is to fill the gap of such knowledge.

Tire/road stick-to-slip transition determines the rubber deformation and friction force distributions. The stick contact refers to no locally relative movement between the tire and the road, while in slip contact, relative tire/road motion exists locally. Studying the static tire/road stick-to-slip transition is important for several reasons. Modeling and sensing the stick-slip interactions will build the knowledge of the rubber deformation and friction force distributions on the contact patch and their evolutions [10]. Understanding these distributions provides the foundation to capture the dynamic tire/road interactions and to build accurate friction models for vehicle dynamic simulation and active 
safety control [11], [12]. Obtaining the local friction forces and their distributions in tire/road interactions is a challenging task, even for the case of static stick-slip interactions. Finally, the knowledge and modeling outcomes of studying static tire/road stick-slip interactions can be potentially extended to study flexible thin-layer/rigid contacts in other mechanical systems, such as robotic grasping applications.

Unlike robotic fingertip contact [13], [14], the Hertzian contact theory [15] cannot be directly used to obtain the tire/road normal contact force distribution due to the large rubber deformation and the tire's hollow structure. A semi-analytical normal force model for the thin layer rigid contact [16] is instead used and extended. To obtain the elastic rubber deformation and friction force distributions simultaneously, a beam-spring network model is introduced and employed. Inspired by the discrete mass-spring network models that are used to successfully simulate and compute the deformation of thin-layer structures [17], [18], a set of virtual cantilever beams are used to model the rubber's shear effect and a set of virtual springs that connect the beams are introduced to capture the elasticity. The cantilever beam-spring modeling approach not only provide the fundamental information of deformation and force distributions, but also fits well with tire/road dynamic friction models such as the LuGre models in [6] and [7]. Therefore, it is naturally convenient to integrate the beam-spring network model with the dynamic tire/road friction model to bridge the local friction force distribution with the total friction force.

In order to focus on the fundamentals of mechanical contact properties, a small motorcycle tire with a smooth outer surface is used in this study. To validate the analyses and the models, an embedded, small sensor made of pressure-sensitive, electric conductive rubber (PSECR) is used to extract the multidirectional local friction forces on the tire contact patch [19]. Compared with the other types of the tire sensors (e.g., [20], [21]), the embedded PSECR sensor is capable to extract the local friction forces and their distributions.

The main contribution of the paper lies in the force and deformation distribution models, analyses, and experiments for studying stick-slip contacts between a flexible thin layer and a rigid surface. These contact problems include not only the tire/road interactions but also many other applications in mechanical systems, such as robotic grasping and manipulation [22], etc. Although the presented work focuses on the static stick-slip interaction, the results provide new modeling approaches to study the dynamic tire/road interactions. The calculation and modeling of the local contact force distributions can also lead to potentially build relationships among the beam-spring network model, the embedded tire sensor measurements, and the total friction models (e.g., LuGre dynamic friction model). These relationships are particularly useful for using the embedded sensors to estimate the model parameters in the total friction models in real time. Comparing with the previously presented conference papers [23], [24], more comprehensive analytical models and extensive experiments are reported here.

The rest of the paper is organized as follows. Section II discusses the model for the tire/road normal contact force. Section III presents the stick-slip interaction model. The experimental setup and results are demonstrated in Section IV.

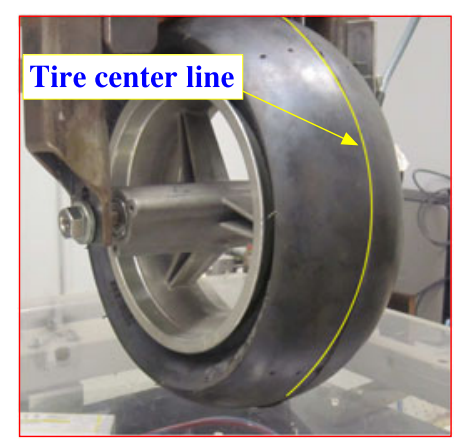

(a)

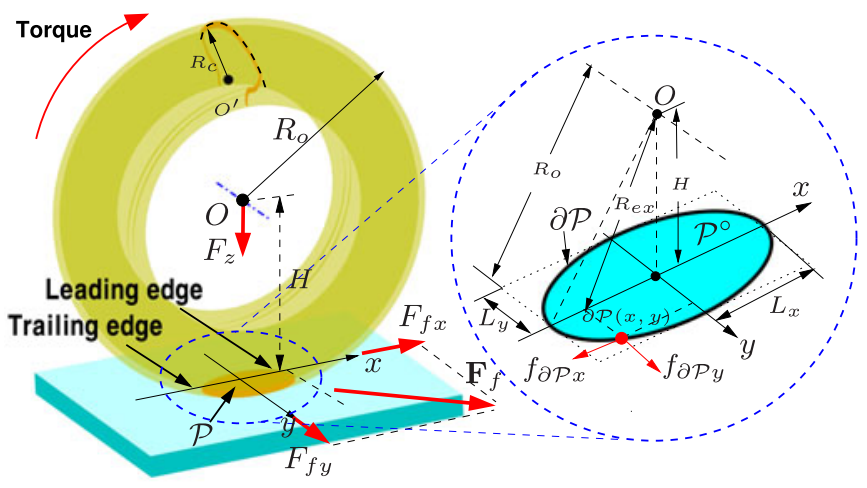

(b)

Fig. 1. (a) Torus-shape tire/road contact. (b) Schematic of the tire/road contact and the boundary force calculation.

Finally, the concluding summary and discussion of the future work are included in Section V.

\section{Tire/RoAd Normal Contact Pressure Model}

\section{A. Tire Contact Patch Geometry}

Fig. 1(a) shows the tire/road contact and Fig. 1(b) illustrates the schematic of the contact geometry and force generation. A torus-shape motorcycle tire with a smooth outer surface and a circular radial cross section is used in this study. A coordinate system is set up with the origin at the center of contact patch $\mathcal{P}$ with a vertical distance of $h$ to the road surface. The $x$-axis is along the tire center with the positive direction toward the leading edge of $\mathcal{P}$, that is, the direction where the tire tends to move forward. Total normal force $F_{z}$ is applied on $\mathcal{P}$ with normal contact pressure distribution $P_{n}(x, y)$ and inflation air pressure $P_{\text {air }}$. Total friction force $\mathbf{F}_{f}$ is projected along the $x$ and $y$-axis directions as $F_{f x}$ and $F_{f y}$, respectively. The friction force stress $\mathbf{P}_{f}(x, y)$ at point $(x, y) \in \mathcal{P}$ is composed as the $x$ and $y$-axis components $P_{f x}(x, y)$ and $P_{f y}(x, y)$, respectively. From the above definitions, it is straightforward to obtain

$$
F_{z}=\int_{\mathcal{P}} P_{n}(x, y) d S, \quad \mathbf{F}_{f}=\int_{\mathcal{P}} \mathbf{P}_{f}(x, y) d S
$$

where $d S$ is an infinitesimal area on $\mathcal{P}$.

Under $P_{\text {air }}$ and $F_{z}, \mathcal{P}$ is in an elliptical shape [23], [25]. To estimate the major radius $L_{x}$ and the minor radius $L_{y}$ of $\mathcal{P}$, a series of experiments are conducted under various $P_{\text {air }}$ and $F_{z}$. An empirical model for $L_{k}, k=x, y$, is used to capture the 


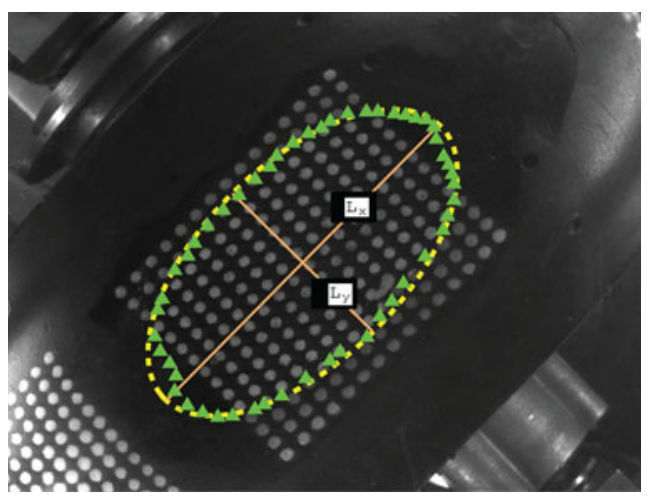

(a)

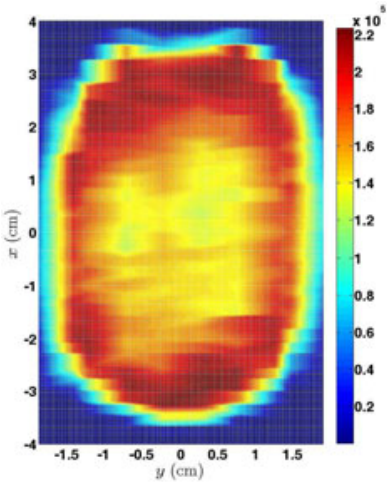

(b)

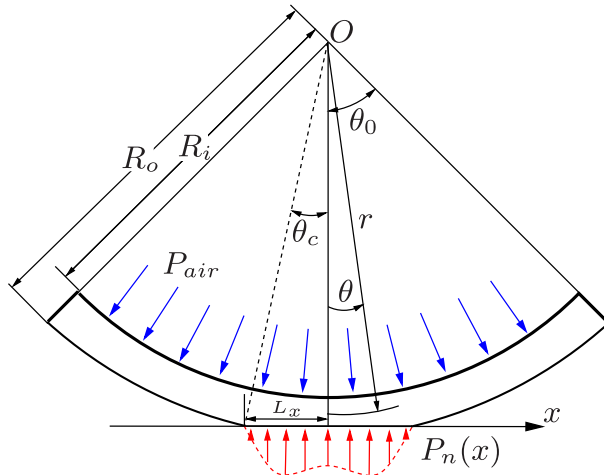

(c)

Fig. 2. (a) Contact patch geometry. The dashed-line indicates the elliptic model approximation and the triangular line shows the actual contact contour from the images taken by a camera underneath the tire. The white dots painted on the tire surface are used to calculate the rubber deformation distribution. (b) $P_{n}(x, y)$ distribution (in Pa) under $P_{\text {air }}=69 \mathrm{kPa}$ and $F_{z}=267 \mathrm{~N}$. (c) Schematic of the tire contact configuration along the center line.

relationships among $L_{k}, F_{z}$, and $P_{\text {air }}$ as follows [23]:

$$
L_{k}\left(P_{\text {air }}, F_{z}\right)=a_{k}\left(P_{\text {air }}\right) F_{z}^{b_{k}\left(P_{\text {air }}\right)}, \quad k=x, y
$$

where $a_{k}\left(P_{\text {air }}\right)=e^{a_{k 2} P_{\text {air }}^{2}+a_{k 1} P_{\text {air }}+a_{k 0}}, b_{k}\left(P_{\text {air }}\right)=b_{k 2} P_{\text {air }}^{2}+$ $b_{k 1} P_{\text {air }}+b_{k 0}$. The values of parameters $a_{k i}$ and $b_{k i}, k=x, y$, $i=1,2,3$, and the validation of (1) are presented in [23]. As an example, the comparison of the model predicted ellipse and the measured $\mathcal{P}$ is shown in Fig. 2(a). The prediction matches the actual size of $\mathcal{P}$. With the known $L_{x}$ and $L_{y}$, the distance between the tire center $O$ and $\mathcal{P}$ is calculated as $H=\sqrt{R_{o}^{2}-L_{x}^{2}}$. From the geometry in Fig. 1(b), the radius of the tire radial cross section is obtained as $R_{c}=\frac{\left(R_{o}-H\right)^{2}+L_{y}^{2}}{2\left(R_{o}-H\right)}$.

\section{B. Normal Contact Pressure Distribution $P_{n}(x, y)$}

Because of its influences on the friction force distribution, the normal contact pressure distribution is discussed in this section. Fig. 2(b) shows a typical normal contact pressure distribution $P_{n}(x, y)$ that is obtained from the measurements of a set of PSECR sensor arrays placed on $\mathcal{P}$. It is clear that $P_{n}(x, y)$ is symmetric with respect to the center of $\mathcal{P}$. Unlike the fingertip contact in which the highest pressure of $P_{n}(x, y)$ is located around the center [14], the locations of the highest $P_{n}(x, y)$ form a ring-shaped zone and are close to the edge of $\mathcal{P}$. In [23], an empirical model is presented for $P_{n}(x, y)$. To provide a better understanding of the normal contact pressure distribution, a semi-analytical model is derived and obtained in this section. To simplify the contact modeling and achieve analytical results, it is assumed that the tire is made of homogeneous rubber without any reinforcing fiber/steel layers.

Due to the symmetry, the analytical model along the tire center line [see Fig. 1(a)] is presented here. Such a 1-D pressure distribution is denoted as $P_{n}(x)$ and Fig. 2(c) illustrates the schematic to calculate $P_{n}(x)$. The Fourier-Michell solution [26] is used to obtain $P_{n}(x)$. To satisfy the biharmonic condition $\nabla^{4} \phi(r, \theta)=0$ in polar coordinates [26], the following potential function $\phi(r, \theta)$ is selected [16]:

$$
\begin{array}{r}
\phi(r, \theta)=a_{0} r^{2}+b_{0} \log r+\sum_{n=1}^{\infty}\left(a_{n} r^{n \pi / \theta_{0}}+b_{n} r^{n \pi / \theta_{0}+2}\right. \\
\left.+c_{n} r^{-n \pi / \theta_{0}}+d_{n} r^{-n \pi / \theta_{0}+2}\right) \cos \frac{n \pi \theta}{\theta_{0}}
\end{array}
$$

where $a_{0}, b_{0}, a_{n}, b_{n}, c_{n}$, and $d_{n}$ are coefficients to be determined and $\theta_{0}$ is defined for the cross section where the contacting influence on deflection and shear stress are negligible. In Fig. 2(c), contact angle $\theta_{c}=\sin ^{-1}\left(\frac{L_{x}}{R_{o}}\right)$. Given the potential function in (2), the radial normal stress $\sigma_{r r}$, the tangential normal stress $\sigma_{\theta \theta}$, and the shear stresses $\tau_{r \theta}$ in polar coordinates are calculated as follows [26]:

$$
\sigma_{r r}=\frac{1}{r} \frac{\partial \phi}{\partial r}+\frac{1}{r^{2}} \frac{\partial^{2} \phi}{\partial \theta^{2}}, \sigma_{\theta \theta}=\frac{\partial^{2} \phi}{\partial r^{2}}, \tau_{r \theta}=-\frac{\partial}{\partial r}\left(\frac{1}{r} \frac{\partial \phi}{\partial \theta}\right) .
$$

For calculation convenience, normalized $\hat{\theta}=\frac{\pi \theta}{\theta_{0}}$ and $\hat{\theta}_{c}=$ $\frac{\pi \theta_{c}}{\theta_{0}}$ are used in the following calculations. Without confusion, the notations are abused by using $\theta$ and $\theta_{c}$ instead of $\hat{\theta}$ and $\hat{\theta}_{c}$ for brevity.

The normalized boundary conditions are set as

$$
\begin{aligned}
& u_{\theta}=\tau_{r \theta}=0,|\theta|=\pi, R_{i} \leq r \leq R_{o} \\
& \sigma_{r r}=\tau_{r \theta}=0,0 \leq|\theta|<\pi, r=R_{i} \\
& \sigma_{r r}=\tau_{r \theta}=0, \theta_{c} \leq|\theta|<\pi, r=R_{o}, \text { and } \\
& \left\{\begin{array}{l}
\frac{\partial u_{r}}{\partial \theta}=\frac{R_{o} \theta_{0}^{2}}{\pi^{2}} \sin \theta, 0 \leq|\theta|<\theta_{c}, r=R_{o} \\
\tau_{r \theta}=0,
\end{array}\right.
\end{aligned}
$$

where $u_{r}$ and $u_{\theta}$ are radial and tangential displacements in polar coordinates, respectively. Notice that directly applying the boundary condition $\sigma_{r r}=-P_{\text {air }}$ at $r=R_{i}$ will not capture the influence of the $P_{\text {air }}$ on the solutions due to the modeling setup and limitation. Instead, the influence of $P_{\text {air }}$ on the normal contact pressure is temporarily not considered in the above model and its effect will be included later.

Plugging (2) and (3) with the normalized $\theta$ into (4), the coefficients in $\phi(r, \theta)$ are solved and obtained. Following the similar derivation in [16], an analytical solution for the normal contact stress at $r=R_{o}$ is obtained as

$$
\sigma_{r r}\left(R_{o}, \theta\right)=\cos \left(\frac{\theta}{2}\right) \int_{\theta}^{\theta_{c}} \frac{\varphi(t) d t}{\sqrt{\cos \theta-\cos t}}
$$


where $\varphi(t)$ is an auxiliary function that is found as a Fredholm integral problem of the second kind

$$
\varphi(t)+\int_{0}^{\theta_{c}} \varphi(s) K(t, s) d s=-\frac{G}{\sqrt{2}(1-\nu)}\left(\frac{\theta_{0}}{\pi}\right)^{2} \sin t
$$

and the kernel function $K(t, s)$ is defined as

$$
\begin{aligned}
K(t, s)=-\frac{1}{2} \sum_{n=1}^{\infty} & n\left(1-\frac{G}{1-\nu} h_{n}\right) \tan \left(\frac{t}{2}\right)\left\{\left[L_{n}(\cos s)\right.\right. \\
& \left.\left.+L_{n-1}(\cos s)\right]\left[L_{n}(\cos t)+L_{n-1}(\cos t)\right]\right\}
\end{aligned}
$$

$L_{n}(x)$ is a Legendre polynomial of order $n$, and $h_{n}$ is defined by (7), as shown at the bottom of the page, $\hat{n}=\frac{n \pi}{\theta_{0}}$, and parameter $\rho=\frac{R_{i}}{R_{o}}$ is the ratio of $R_{i}$ and $R_{o}$.

Considering $\theta$ in (5) actually represents $\hat{\theta}$, substituting $\hat{\theta}=\frac{\pi \theta}{\theta_{0}}$ into (5) and taking a small angle assumption for $\theta$, $\sigma_{r r}\left(R_{o}, \frac{\pi x}{\theta_{0} R_{o}}\right)$ is then used to approximate $P_{n}(x)$ at $x=R_{o} \theta$.

Remark 1: Although the distribution of $\sigma_{r r}\left(R_{o}, \frac{\pi x}{\theta_{0} R_{o}}\right)$ only along the tire center line is presented, the results are readily extended to the distribution along the $y$-axis direction because the radial cross section is also in a circular shape. Similar to the approach presented in [23], the 2-D model of $\sigma_{r r}(x, y)$ distribution on $\mathcal{P}$ can be obtained.

To capture the influence of $P_{\text {air }}$, an empirical approach is considered. For a given $F_{z}$, the semi-analytical model of normal contact pressure $P_{n}(x, y)$ is calculated as

$$
P_{n}(x, y)=-\sigma_{r r}(x, y)+\frac{1}{S_{\mathcal{P}}}\left\{F_{z}-\int_{\mathcal{P}}\left[-\sigma_{r r}(x, y)\right] d S\right\}
$$

where $S_{\mathcal{P}}=\pi L_{x} L_{y}$ is the total area of $\mathcal{P}$ and the negative sign of $\sigma_{r r}(x, y)$ follows the convention that a compressive stress is defined as a negative value. The second term in (8) captures a uniform pressure distribution on $\mathcal{P}$ induced by inlet tire pressure $P_{\text {air }}$. The effectiveness of such a treatment will be validated through experiments in Section IV.

Remark 2: The above derivation does not consider the existence of the friction force $\mathbf{F}_{f}$ on $\mathcal{P}$. The experimental results show that during the stick-to-slip transition, the peak values of $P_{n}(x, y)$ tend to shift toward the leading edge. However, in contrast to the fingertip contact [13], [14], [27], the magnitude of change of $P_{n}(x, y)$ during stick-to-slip transitions is limited due to the hollow structure and the relatively smaller friction forces than those in dynamic interactions of rotating tires [8]. Therefore, the change of $P_{n}(x, y)$ under $\mathbf{F}_{f}$ is neglected in the rest of discussions.

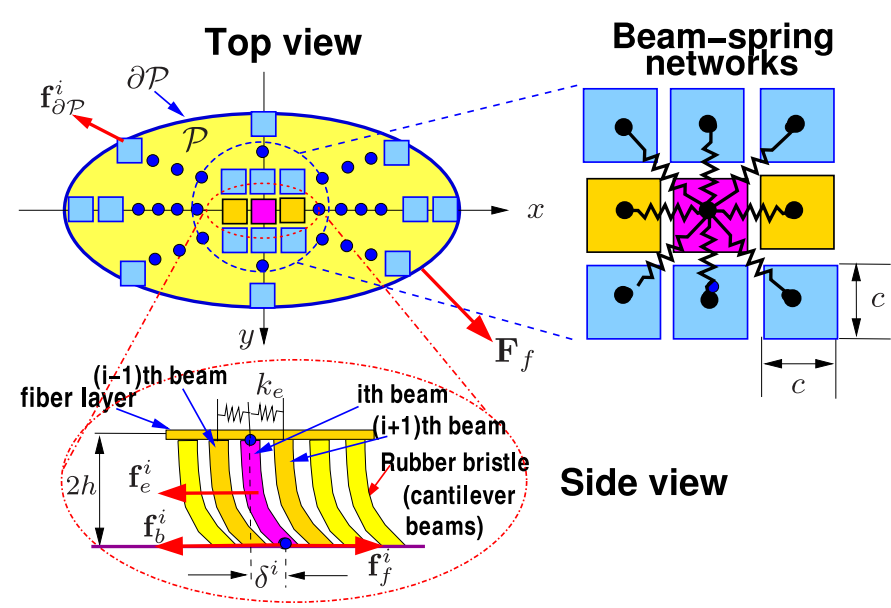

Fig. 3. Schematic of the hybrid beam-spring network model to capture tire/road stick-slip interaction.

\section{STICK-SLiP ANALYSIS, Modeling, AND SENSING}

\section{A. Beam-Spring Network Modeling}

During the stick-to-slip transition, some contact points on $\mathcal{P}$ begin deforming and slipping while other points remain sticking to the road. The slipping region grows as the applied force increases. It is difficult to obtain a closed-form formulation of the deformation and force distributions. Instead, a beam-spring network approach is used to compute the local deformation and the friction force distributions simultaneously.

Fig. 3 illustrates the beam-spring network modeling approach. The contact patch $\mathcal{P}$ is partitioned into $N$ virtual cantilever beams and each of them has a height of $2 h$ and a square cross section with dimension $c \times c$, where $c$ is the side length. Virtual linear springs connect each pair of neighboring beams. The following assumptions are considered: 1) for each beam, elongation and compression are ignored and only the bending deformation is considered, and 2) all the cantilever beams are clamped at one end through the tire inlet fiber layer. The fiber layer is flexible to allow deformation. Although the springs connect each pair of neighboring beams, there is no spatial gap among them.

Let $\mathbf{I}_{\mathcal{P} \circ}$ and $\mathbf{I}_{\partial \mathcal{P}}$ denote the index sets of the beams in $\mathcal{P}^{\circ}$ and on $\partial \mathcal{P}$, respectively. During stick-to-slip transition, dynamic motions of the beams are negligible. For the $i$ th beam, $i=$ $1, \ldots, N$, the following force equilibrium is obtained:

$$
\begin{cases}\mathbf{f}_{\mathbf{f}}^{\mathrm{i}}+\mathbf{f}_{\mathbf{b}}^{\mathbf{i}}+\mathbf{f}_{\mathbf{e}}^{\mathbf{i}}=\mathbf{0}, & i \in \mathbf{I}_{\mathcal{P}^{\circ}} \\ \mathbf{f}_{f}^{i}+\mathbf{f}_{b}^{i}+\mathbf{f}_{e}^{i}+\mathbf{f}_{\partial \mathcal{P}}^{i}=0, & i \in \mathbf{I}_{\partial \mathcal{P}}\end{cases}
$$

where $\mathbf{f}_{f}^{i}$ is the tire contact friction force, $\mathbf{f}_{b}^{i}$ is the bending force that captures the shear deformation, $\mathbf{f}_{e}^{i}$ is the resultant net elastic force from the connected springs, and $\mathbf{f}_{\partial \mathcal{P}}^{i}$ is the boundary force from the tire side wall if the beam is on $\partial \mathcal{P}$; see Fig. 3. In the

$$
h_{n}=\frac{\hat{n}\left[(2 \nu-1)\left(\rho^{2 \hat{n}}-1\right)^{2}+2 \hat{n}(\nu-1)\left(\rho^{4 \hat{n}}-1\right)-\hat{n}^{2} \rho^{2 \hat{n}-2}\left(\rho^{2}-1\right)\left(\rho^{2}+3-4 \nu\right)\right]}{2 G\left(\hat{n}^{2}-1\right)\left[\left(\rho^{2 \hat{n}}-1\right)^{2}-\hat{n}^{2} \rho^{2 \hat{n}-2}\left(\rho^{2}-1\right)^{2}\right]}
$$


following, the calculations of $\mathbf{f}_{b}^{i}, \mathbf{f}_{e}^{i}$, and $\mathbf{f}_{\partial \mathcal{P}}^{i}$ are discussed such that $\mathbf{f}_{f}^{i}$ is obtained by (9).

Because of relative movements among beams, the resultant elastic force $\mathbf{f}_{e}^{i}$ is calculated as

$$
\mathbf{f}_{e}^{i}=\sum_{j \in \mathcal{N}_{i}} \mathbf{f}_{e}^{i j}=\sum_{j \in \mathcal{N}_{i}} k_{e} \Delta \mathbf{u}_{i j}=2 E h \sum_{j \in \mathcal{N}_{i}} \Delta \mathbf{u}_{i j}
$$

where $\mathbf{f}_{e}^{i j}=k_{e} \Delta \mathbf{u}_{i j}$ is the spring (elastic) force between the $i$ th beam with its neighboring $j$ th beam, $k_{e}=2 E h$ is the spring stiffness coefficient, and $\mathcal{N}_{i}$ is the index set of all the neighboring beams of the $i$ th beam. Spring deformation

$$
\Delta \mathbf{u}_{i j}=\left(\mathbf{u}_{j}-\mathbf{u}_{i}\right)\left(1-\frac{\left\|\mathbf{u}_{j 0}-\mathbf{u}_{i 0}\right\|}{\left\|\mathbf{u}_{j}-\mathbf{u}_{i}\right\|}\right)
$$

is the relative position change between the $i$ th and $j$ th beams, where $\mathbf{u}_{i}$ and $\mathbf{u}_{j}$ are the position vectors for the $i$ th and $j$ th beams, respectively. In (11), $\mathbf{u}_{i 0}$ and $\mathbf{u}_{j 0}$ are, respectively, the original position vectors of the $i$ th and $j$ th beams without deformation. Indeed, it is straightforward to obtain

$\left\|\mathbf{u}_{j 0}-\mathbf{u}_{i 0}\right\|= \begin{cases}\sqrt{2} c, & \text { Beams } i \text { and } j \text { are diagonal neighbors } \\ c, & \text { otherwise. }\end{cases}$

The virtual cantilever beams are bent under $\mathbf{F}_{f}$. The tip bending deformation $\delta_{k}^{i}$ of the $i$ th beam along the $k$-axis is approximated by [13]

$$
\delta_{k}^{i}=\frac{P_{n}^{i}}{P_{n}^{\text {ave }}} \frac{3 \mu F_{z}}{16 L_{k}} \frac{2-\nu}{G}\left[1-\left(1-\psi_{k}\right)^{2 / 3}\right], k=x, y
$$

where $\mu$ is the friction coefficient, $P_{n}^{i}$ is the local normal pressure at the $i$ th beam's location, $P_{n}^{\text {ave }}$ is the average normal pressure of $\mathcal{P}$, and $\psi_{k}=\frac{F_{f k}}{\mu F_{z}}$ is the friction force factor in the $k$-axis direction. Comparing with the model in [13], a normal pressure dependency factor $\frac{P_{n}^{i}}{P_{n}^{n v e}}$ is introduced in (12) to capture the effect of $P_{n}(x, y)$ on $\delta_{k}^{i}$. The bending force of the $i$ th beam is then obtained as

$$
\mathbf{f}_{b}^{i}=k_{b} \boldsymbol{\delta}^{i}=\frac{3 E I}{(2 h)^{3}} \boldsymbol{\delta}^{i}
$$

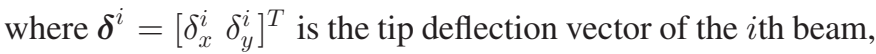
and $k_{b}=\frac{3 E I}{(2 h)^{3}}$ is the bending stiffness of the beam.

To compute $\mathbf{f}_{f}^{i}$ for $i \in \mathbf{I}_{\mathcal{P}^{\circ}}$, the Coulomb friction model is adopted. If $\left|\mathbf{f}_{b}^{i}+\mathbf{f}_{e}^{i}\right|<\mu f_{z}^{i}$, where $f_{z}^{i}=P_{n}^{i} c^{2}$ is the normal force at the tip of the $i$ th beam, the beam is stuck and has no movement. In this case, $\mathbf{f}_{f}^{i}$ balances the resultant of $\mathbf{f}_{b}^{i}$ and $\mathbf{f}_{e}^{i}$. If $\left|\mathbf{f}_{b}^{i}+\mathbf{f}_{e}^{i}\right| \geq \mu f_{z}^{i},\left|\mathbf{f}_{f}^{i}\right|=\mu f_{z}^{i}$ cannot fully sustain the resultant of $\mathbf{f}_{b}^{i}$ and $\mathbf{f}_{e}^{i}$. The beam will slip until the force equilibrium holds again at the new location. $\mathbf{f}_{b}^{i}$ is assumed to be saturated when the $i$ th beam slips.

Remark 3: The eight-neighboring-beam topology and the beam's square cross section shown in Fig. 3 are chosen because under these configurations, the model represents a structure with an equivalent Poisson's ratio around 0.5 [17], almost the same as the value of rubber $\nu=0.49$. The number of beams $N$ is important for an accurate beam-spring network model. With a larger $N$, the model predictions are more accurate [17].
Remark 4: In both the normal contact pressure model and the stick-slip interaction model, linear elastic assumption is used. The effectiveness of linear elastic assumption is approximately verified by the tensile experiments using the tire rubber specimens. As shown in the later sections, the consistent comparison results between the model predictions and the sensor measurements confirm the linear elastic model approximation. Moveover, the beam-spring scheme is readily extendable to incorporate any rubber nonlinear elastic models by modifying (10).

In the above discussion, only the interior beams in $\mathcal{P}^{\circ}$ are considered. For the beams on $\partial \mathcal{P}$, the same calculations are followed but with additional consideration for boundary force $\mathbf{f}_{\partial \mathcal{P}}^{i}$, which is discussed in the next section.

\section{B. Calculation of Boundary Force $\mathbf{f}_{\partial \mathcal{P}}^{i}$}

By adding all $N$ equations in (9) together, the total boundary force $\mathbf{F}_{\partial \mathcal{P}}=\sum_{i \in \mathbf{I}_{\partial \mathcal{P}}} \mathbf{f}_{\partial \mathcal{P}}^{i}$ is obtained as

$$
\mathbf{F}_{\partial \mathcal{P}}=-\mathbf{F}_{b}-\mathbf{F}_{f}
$$

where the total bending force $\mathbf{F}_{b}$ is

$$
\mathbf{F}_{b}=\sum_{i=1}^{N} \mathbf{f}_{b}^{i} \text { and } \mathbf{F}_{f}=\sum_{i=1}^{N} \mathbf{f}_{f}^{i} .
$$

To obtain (14), the fact that total spring force $\mathbf{F}_{e}=\sum_{i=1}^{N} \mathbf{f}_{e}^{i}=\mathbf{0}$ is used since $\mathbf{f}_{e}^{i}$ s are internal spring forces and their summation is zero.

The extension part of Fig. 1(b) illustrates the schematic of the calculation of boundary force $\mathbf{f}_{\partial \mathcal{P}}^{i}$ of the $i$ th beam at location $(x, y)$ on $\partial \mathcal{P}$. For a given $\mathbf{F}_{f}$ and $F_{z}, \mathbf{F}_{b}$ is calculated by (13) and (15) and $\mathbf{F}_{\partial \mathcal{P}}$ is then obtained from (14). $\mathbf{F}_{\partial \mathcal{P}}$ is further decomposed along the $x$ - and $y$-axis directions as $F_{\partial \mathcal{P} x}$ and $F_{\partial \mathcal{P} y}$, respectively. In the following, only the calculation of the $x$-axis component force $f_{\partial \mathcal{P} x}^{i}$ of $\mathbf{f}_{\partial \mathcal{P}}^{i}$ is described and $f_{\partial \mathcal{P} y}^{i}$ can be obtained similarly. Boundary force $f_{\partial \mathcal{P} x}^{i}$ is generated through rubber deformation. By symmetry, it is assumed that $F_{\partial \mathcal{P}_{x}}$ is equally distributed on each quarter of the elliptical boundary such that only one quarter of $\partial \mathcal{P}$ with a total boundary force $\frac{1}{4} F_{\partial \mathcal{P} x}$ is considered.

For the boundary point $(x, y)$ shown in Fig. 1(b), the radius $R_{e x}$ with respect to rotation axis is obtained as $R_{e x}=$ $\sqrt{H^{2}+x^{2}}$. During the stick-to-slip transition, it is assumed that the entire tire/rim structure rotates about an infinitesimal angle $\Delta \theta$. The projected displacement along $\partial \mathcal{P}$ in the $x y$ plane is $\Delta x=R_{e x} \Delta \theta \cos \theta \approx R_{e x} \Delta \theta$ for a small $\theta$. Letting $K_{s}$ denote the rubber stiffness coefficient, under deformation $\Delta x$, boundary force $f_{\partial \mathcal{P} x}^{i}$ is then

$$
f_{\partial \mathcal{P} x}^{i}=K_{s} \Delta x=K_{s} \Delta \theta \sqrt{H^{2}+x^{2}} .
$$

Considering that the total boundary force on a quarter of $\partial \mathcal{P}$ is $\frac{1}{4} F_{\partial \mathcal{P} x}$, it is straightforward to have

$$
\frac{1}{4} F_{\partial \mathcal{P} x}=\int_{\partial \mathcal{P}_{1}} f_{\partial \mathcal{P}_{x}} d s=\int_{\partial \mathcal{P}_{1}} K_{s} \Delta \theta \sqrt{H^{2}+x^{2}} d s
$$




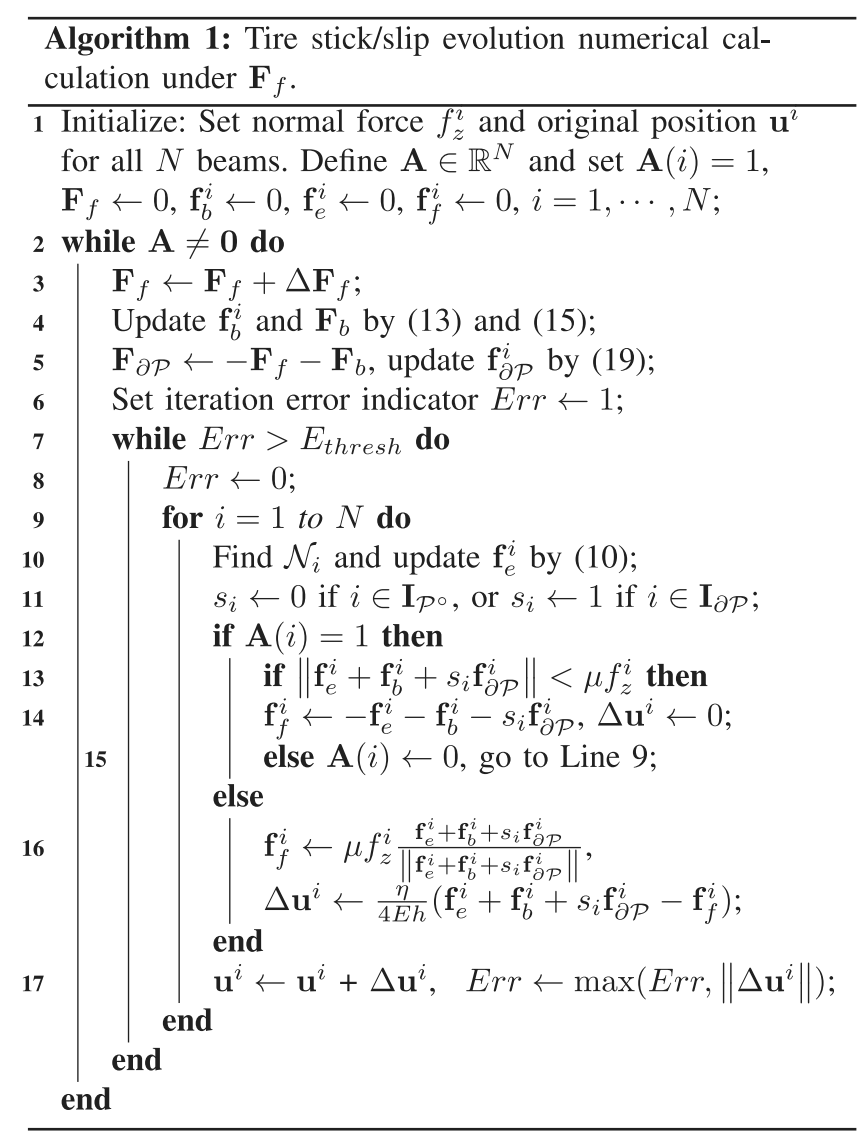

where $s$ is the arc-length and $\partial \mathcal{P}_{1}$ is the quarter of $\partial \mathcal{P}$. Considering that $(d s)^{2}=(d x)^{2}+(d y)^{2}$ and that $d y=-\frac{x L_{y}^{2}}{y L_{x}^{2}} d x$ from the elliptical equation $\frac{x^{2}}{L_{x}^{2}}+\frac{y^{2}}{L_{y}^{2}}=1$, (17) becomes

$$
\begin{aligned}
\frac{1}{4} F_{\partial \mathcal{P} x} & =\int_{0}^{L_{x}} K_{s} \Delta \theta \sqrt{H^{2}+x^{2}} \sqrt{1+\left(\frac{x L_{y}^{2}}{y L_{x}^{2}}\right)^{2}} d x \\
& =K_{s} \Delta \theta K_{x}
\end{aligned}
$$

where

$$
K_{x}=\int_{0}^{L_{x}} \sqrt{H^{2}+x^{2}} \sqrt{1+\frac{L_{y}^{2} x^{2}}{L_{x}^{2}\left(L_{x}^{2}-x^{2}\right)}} d x .
$$

In the last step of (18), the fact that $L_{x}^{2} y^{2}=L_{y}^{2}\left(L_{x}^{2}-x^{2}\right)$ is used to obtain $K_{x}$. Plugging the results in (18) into (16), the final calculation for $f_{\partial \mathcal{P} x}^{i}$ is obtained as

$$
f_{\partial \mathcal{P} x}^{i}=\frac{\sqrt{H^{2}+x^{2}}}{4 K_{x}} F_{\partial \mathcal{P} x} .
$$

\section{Computing the Deformation and Friction Force Distributions}

Using the beam-spring network model, Algorithm 1 illustrates iteratively computing of deformation distribution $\mathbf{u}$ and friction force $\mathbf{f}_{f}$ on $\mathcal{P}$ simultaneously. In the algorithm, a variable $\mathbf{A} \in \mathbb{R}^{N}$ is defined to update the stick $(\mathbf{A}(i)=1)$ or the $\operatorname{slip}(\mathbf{A}(i)=0)$ status for the $i$ th beam. An error threshold $E_{\text {thresh }}>0$ is also introduced to terminate the iterations.

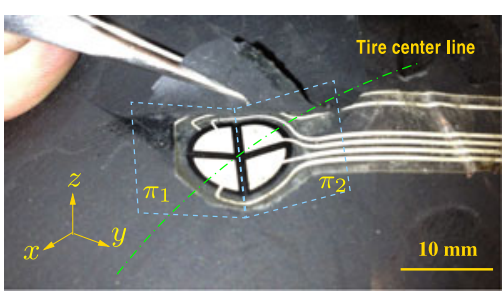

(a)

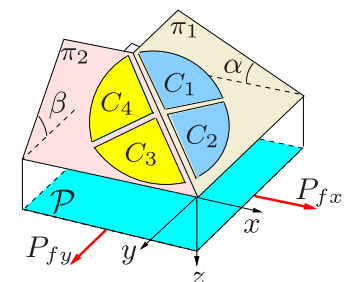

(b)
Fig. 4. Embedded PSECR sensor configuration. (a) PSECR sensor embedded inside the tire. (b) Configuration diagrams of the four sensor cells.

An updating factor $0<\eta<1$ is used to tune the convergence performance. The computation follows the calculations discussed previously and the complexity of the algorithm is $O\left(\frac{1}{\eta} N^{2} \log \left(\frac{1}{E_{\text {thresh }}}\right)\right)$. Although a Coulomb friction model is used in the algorithm, it can be replaced by other types of friction models to determine the stick-to-slip transition for each beam.

\section{Embedded Tire Force Sensor}

In this section, measuring the local friction forces on $\mathcal{P}$ by the PSECR sensor is briefly described. The detailed discussion about the sensor model and configuration is presented in [19].

The tire rubber layer is cut and the PSECR sensor is glued and embedded inside the rubber; see Fig. 4(a). The cutting and gluing process is carefully conducted such that minimum destructive effects are made upon the tire structure. The sensor is embedded at the center line of $\mathcal{P}$. The PSECR sensor has four independent sensing cells, denoted as $C_{l}, l=1, \ldots, 4$, and each of them produces a voltage output proportional to the applied normal force on the sensor surface. To obtain the multidirectional force measurements, the sensor is embedded with a special orientation. By tilting the sensor at a certain orientation, the existence of the internal compressive normal stress also prevents the sensor from exposing to elongation forces [19].

It is assumed that the PSECR sensor is small enough such that the local normalized friction force $P_{f x}$ and $P_{f y}$ at the sensor location are uniform; see Fig. 4(b). $P_{f x}$ and $P_{f y}$ are considered as the $x$ - and $y$-axis components of the spatially normalized friction force $\mathbf{f}_{f}^{i}$ in (9). Sensor cells $C_{1}$ and $C_{2}$ are on plane $\pi_{1}$ for measuring $P_{f x}$ while $C_{3}$ and $C_{4}$ are on plane $\pi_{2}$ for measuring $P_{f y}$. Only the calculation of $P_{f x}$ from the measurements of $C_{1}$ and $C_{2}$ is described here, and the same approach is applied to $C_{3}$ and $C_{4}$ to obtain $P_{f y}$.

The sensor model is intertwined with the beam-spring model. The shear stress and the normal stress along the $x$-axis direction (inside the rubber layer) are denoted as $\tau_{x}$ and $\sigma_{x}$, respectively. The embedded sensor cells $C_{1}$ and $C_{2}$ are sensitive to $\tau_{x}$ and $\sigma_{x}$. The distribution of $\sigma_{x}$ is uniform along the $z$-axis direction, while the distribution of $\tau_{x}$ is not, i.e., $\sigma_{x}^{1}=\sigma_{x}^{2}=\sigma_{x}$ and $\tau_{x}^{1} \neq$ $\tau_{x}^{2}$ for $C_{1}$ and $C_{2}$ [19]. In the beam-spring model, let $\sigma_{\mathcal{P} x}$ and $\tau_{\mathcal{P} x}$ denote the spatially normalized $\mathbf{f}_{e}^{i}$ and $\mathbf{f}_{b}^{i}$ along the $x$-axis direction, respectively. By spatially normalizing (9), the magnitude of the normalized friction forces along the $x$-axis direction is thus obtained as

$$
P_{f x}=\tau_{\mathcal{P} x}+\sigma_{\mathcal{P} x}
$$


Considering that spring and beam represent shear effect and normal stain effect, respectively, (20) reveals that $P_{f x}$ actually consists of two parts: $\tau_{\mathcal{P} x}$ corresponds to shear stress $\tau_{x}$ and $\sigma_{\mathcal{P} x}$ is related to the change of normal stress $\sigma_{x}$ along $x$-axis. To obtain $P_{f x}$ by the PSECR sensor, relationships between $\tau_{x}^{1}$, $\tau_{x}^{2}, \sigma_{x}$ and $\tau_{\mathcal{P} x}, \sigma_{\mathcal{P} x}$ are needed. By the beam-spring model, $\tau_{\mathcal{P} x}$ is considered as the boundary stress at the tip of the cantilever beam and $\tau_{x}^{1}$ and $\tau_{x}^{2}$ are the shear stresses at $C_{1}$ and $C_{2}$ locations of the beam. Therefore, linear relationships between $\tau_{\mathcal{P} x}$ and $\tau_{x}^{1}$ and $\tau_{x}^{2}$ are built by using the Euler beam theory and calibration in experiments. Finally, an empirical relationship is proposed to calculated $\sigma_{\mathcal{P} x}$ from $\sigma_{x}$ [19]. If the sensor lies in the stick region, the following relationship is adopted:

$$
\sigma_{\mathcal{P} x}=\kappa_{x}\left|\sigma_{x}\right|^{m_{x}} .
$$

The absolute value of $\sigma_{x}$ in (21) is used because $\sigma_{x}$ can be negative and $\sigma_{\mathcal{P} x}$ is always positive. Coefficients $\kappa_{x}$ and $m_{x}$ are obtained by experimental calibration [19]. When the sensor lies in the location where the local contact slips, $\sigma_{\mathcal{P} x}$ saturates at the value $\sigma_{\mathcal{P} x}^{0}$. Slipping is detected when $\tau_{\mathcal{P} x}$ is saturated and at this moment, $\sigma_{x}=\sigma_{x}^{0}$. Then, $\sigma_{\mathcal{P} x}^{0}=\kappa_{x}\left|\sigma_{x}^{0}\right|^{m_{x}}$ is calculated by (21) for the given $\sigma_{x}^{0}$.

Summarizing the above discussion, it is straightforward to obtain

$$
P_{f x}= \begin{cases}\tau_{\mathcal{P} x}+\sigma_{\mathcal{P} x} & \text { locally stick } \\ \tau_{\mathcal{P} x}+\sigma_{\mathcal{P} x}^{0} & \text { locally slip }\end{cases}
$$

where $\tau_{\mathcal{P} x}$ and $\sigma_{\mathcal{P} x}$ are, respectively, obtained by the calibrated PSECR sensor with the linear relationship between $\tau_{\mathcal{P} x}$ and $\tau_{x}$ and (21).

\section{EXPERIMENTS}

\section{A. "Smart Tire” Test Platform}

Fig. 5 shows the "smart tire" test platform developed at Rutgers University. A treadmill is modified to act as a support foundation for the testing tire. The treadmill belt is removed and replaced by a thick transparent plate to represent the road surface. The plate is supported by three individually computer-controlled electric jacks. Three cable potentiometers (from Celesco Transducer Products, Inc.) are used to feed back the positions and the tilting angles of the plate. Three load cells (from Transducer Techniques Inc.) are positioned under the supporting plate to measure the total normal load between the tire and the plate. A feedback controller is designed to take the load cell's readings and regulate the normal load by raising/lowering the motorized jacks. To generate friction forces along the $x$ - and $y$-axis directions, the tire and the plate are, respectively, pulled by two steel cables: one for the longitudinal ( $x$-axis) direction and the other for the lateral ( $y$-axis) direction. The total friction forces $F_{f x}$ and $F_{f y}$ are measured, respectively, by two force sensors (from Transducer Technique Inc.) as shown in Fig. 5.

The PSECR sensor (from Pongpara Codan Rubber Technology Company, Thailand) is shown in Fig. 5(c). The circular PSECR sensor is around $12 \mathrm{~mm}$ in diameter and is flexible. To measure the tire rubber deformation on the contact patch, a high-resolution camera (CM-080GE camera from Teledyne DALSA Inc., $1032 \times 778$ pixels, 30 frames/s) is used to capture

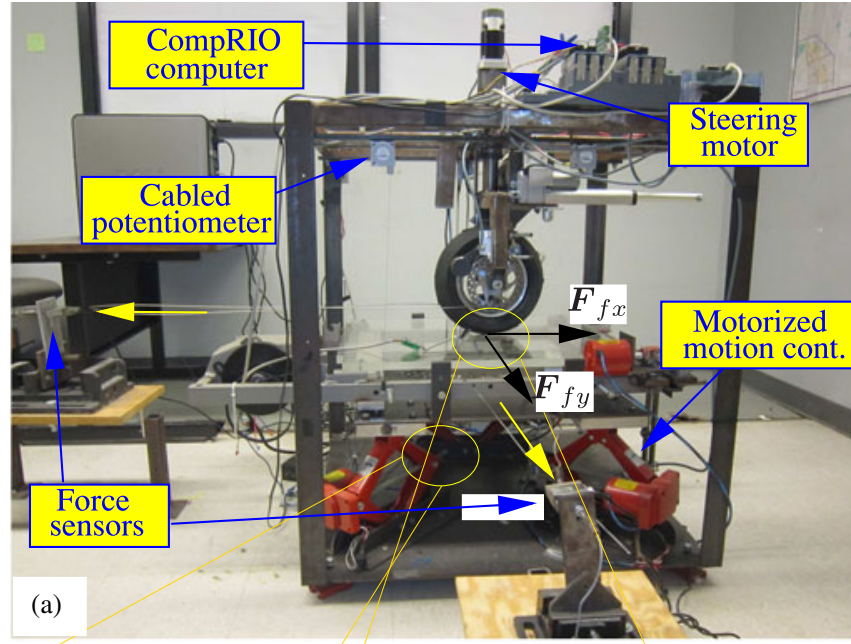

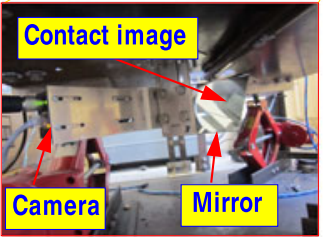

(b)

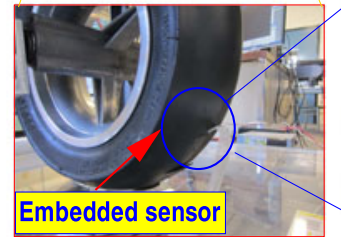

(c)

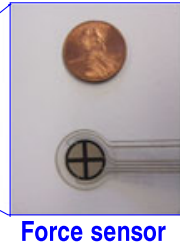

Force sensor
Fig. 5. "Smart tire" test platform prototype with embedded force sensors and vision-based tire deformation measurement systems. (a) Entire system. (b) Camera system setup for deformation measurement. (c) Embedded tire force sensor.

TABLE I

MODEL PARAMETERS

\begin{tabular}{|c|c|c|c|c|c|c|c|c|}
\hline \hline$E(\mathrm{MPa})$ & $G(\mathrm{MPa})$ & $\nu$ & $\mu$ & $\theta_{0}(\mathrm{deg})$ & $R_{o}(\mathrm{~m})$ & $c(\mathrm{~cm})$ & $h(\mathrm{~cm})$ & $N$ \\
\hline 11.7 & 3.9 & 0.49 & 0.7 & 45 & 0.128 & 0.2 & 0.35 & 591 \\
\hline
\end{tabular}

images of white dots [see Fig. 2(a)] painted on the tire surface through the transparent supporting plate; see Fig. 5(b). The image acquisition, camera calibration, and image processing are implemented through the National Instrument (NI) Vision Development Module. The entire system is controlled through a real-time NI CompactRIO embedded system. More details about the setup can be found in [23].

\section{B. Tire Normal Contact Pressure Experiments}

A set of the PSECR sensors are placed on the treadmill surface to measure $P_{n}(x)$ along the tire center line. Fig. 6 shows comparison results between the model prediction and the experimental data. The values of the model parameters are listed in Table I. The value of parameter $\theta_{0}$ cannot be directly measured and is obtained empirically. The model predictions match the experiments under various $P_{\text {air }}$ and $F_{z}$. It is noted that although the simple torus-shape tire is used in this study, as shown in Fig. 6 and in [23], the normal contact pressure distribution follows the similar pattern as those obtained by using actual automobile tires reported in [8] and [9].

One advantage of the analytical model over the empirical model in [23] is that the former can be used to predict $P_{n}(x)$ under variations of physical parameters, while the empirical 


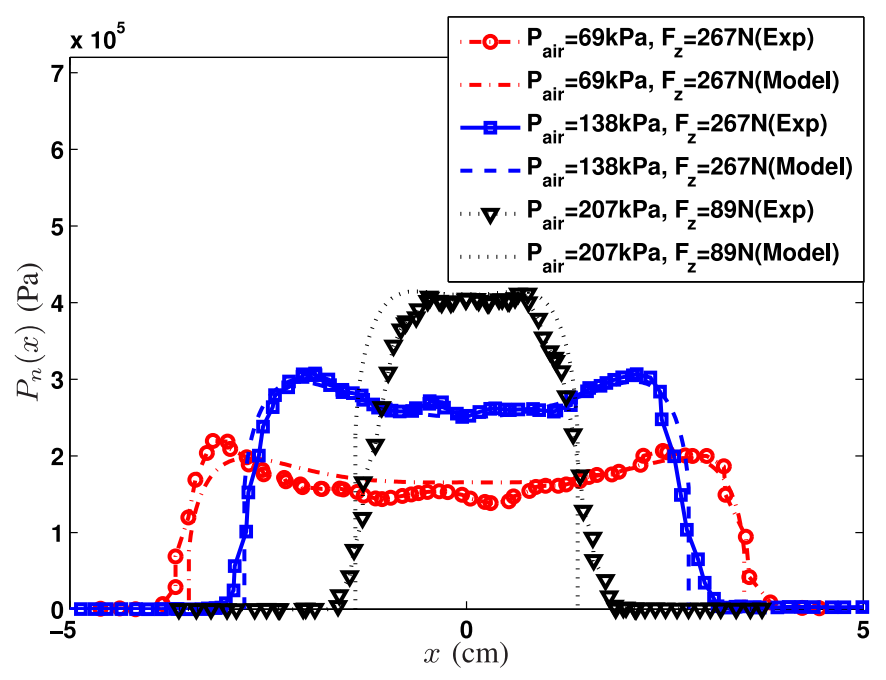

Fig. 6. Comparison of the model prediction and experiments of the tire/road normal contact pressure distribution $P_{n}(x)$.

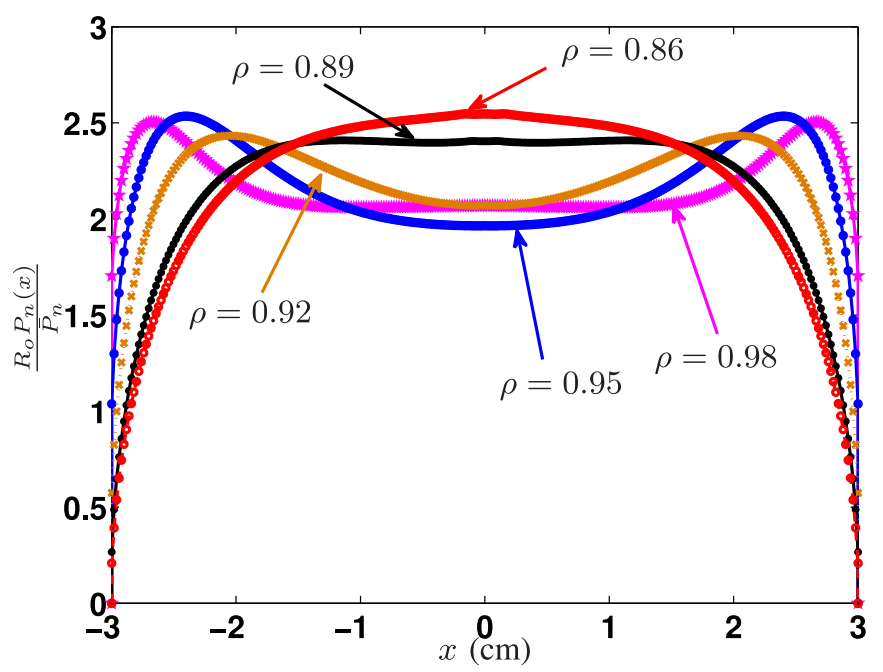

Fig. 7. Normalized contact pressure distribution $P_{n}(x)$ with respect to $\bar{P}_{n}=$ $\int_{-L_{x}}^{L_{x}} P_{n}(x) d x$ under various tire rubber layer thicknesses $\rho, P_{\text {air }}=69 \mathrm{kPa}$.

model cannot. For example, Fig. 7 shows the normalized $P_{n}(x)$ under varying tire rubber layer thicknesses. For tires with a thin rubber layer, $\rho=R_{i} / R_{o} \rightarrow 1$ and a thick layer, $\rho$ is small. It is clearly shown in Fig. 7 that for tires with thin rubber layers (e.g., $\rho>0.9$ ), the bending effect dominates the pressure distribution and the pressure peaks lie close to the contact edge, while for tires with thick rubber layers (e.g., $\rho=0.86), P_{n}(x)$ follows the Hertzian contact with the highest pressure around the center of $\mathcal{P}$. These results explain the observed differences of $P_{n}(x)$ between the tire/road contact and the fingertip contact reported in [13], [14], and [27].

\section{Stick-to-Slip Transition Experiments}

To observe stick-to-slip transition and friction force evolutions on $\mathcal{P}$, the tire is rotated and the sensor is placed at the location around the middle point between the trailing edge and the center of $\mathcal{P}$. An increasing $F_{f x}$ is generated until the tire com-

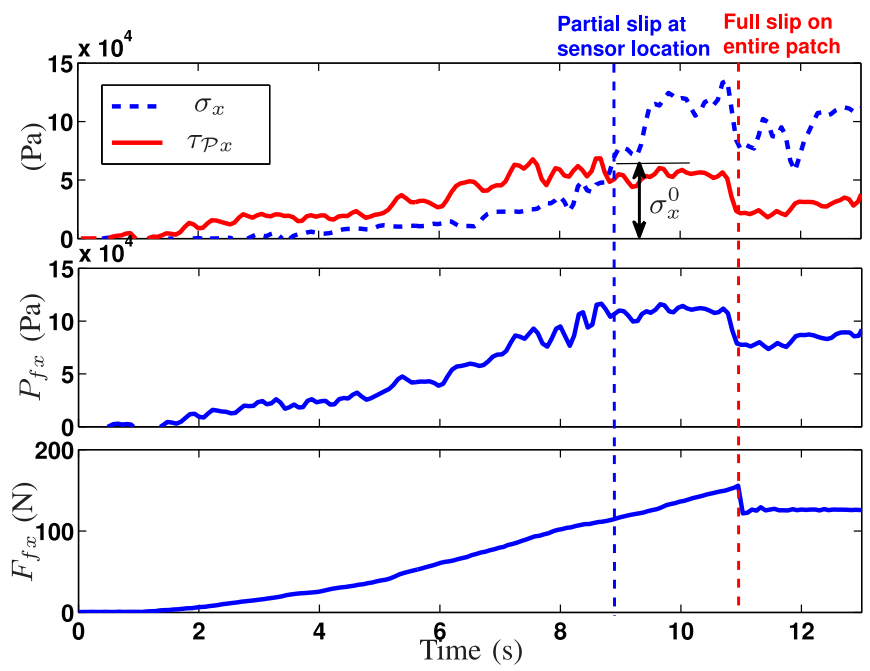

Fig. 8. PSECR sensor measurements and stress estimation at the sensor location during the stick-to-slip transition.

pletely slips (around $F_{f x}=160 \mathrm{~N}$ ). Fig. 8 shows the local friction force measurements at the PSECR sensor location during the stick-to-slip transition. $\tau_{\mathcal{P} x}$ starts to increase with the increasing $F_{f x}$. Once the partial slip at the sensor location starts around $t=8.8 \mathrm{~s}, \tau_{\mathcal{P} x}$ stops increasing and keeps constant. This observation agrees with the previously discussed friction model in which $P_{f x}$ saturates when the partial slip starts. The observation of the saturated $\tau_{\mathcal{P} x}$ is used to predict the moment when the partial slip happens. The value for the estimated $\sigma_{x}^{0}$ is $\sigma_{x}^{0}=6 \times 10^{4} \mathrm{~Pa}$ and, therefore, $\sigma_{\mathcal{P} x}^{0}=\kappa_{x}\left|\sigma_{x}^{0}\right|^{m_{x}}=5.27 \times 10^{4} \mathrm{~Pa}[19]$.

After the partial slip starts, $\sigma_{x}$ keeps increasing with the increasing $F_{f x}$ and the slip region moves towards the interior area of $\mathcal{P}$. The middle plot of Fig. 8 shows the local normalized friction force $P_{f x}$ from the calibrated PSECR sensor. When the full slip of the entire patch starts around $t=11 \mathrm{~s}, \tau_{\mathcal{P} x}$ and $P_{f x}$ drop rapidly. The timing $(t=8.8 \mathrm{~s})$ to determine the partial slip depends on the relative sensor location on $\mathcal{P}$, while the timing of the full slip of $\mathcal{P}(t=11 \mathrm{~s})$ is determined by the magnitude of $F_{f x}$. These observations fit the previously discussed analysis.

\section{Rubber Deformation and Friction Force Distributions}

The experiments of obtaining rubber deformation and friction force distributions are conducted under $P_{\text {air }}=69 \mathrm{kPa}$ and $F_{z}=$ $267 \mathrm{~N}$. The choice of $69 \mathrm{kPa}$ is to prevent the damage of the embedded PSECR sensor and the choice of $267 \mathrm{~N}$ total normal force is to generate a large contact patch. The camera image is used to obtain the rubber deformation on $\mathcal{P}$. Fig. 9 shows the rubber deformation distribution results under $F_{f x}$. In the figure, the blue dots indicate the sticking points and the red bars represent the displacements of slipping points on $\mathcal{P}$. Both the vision-based experiments and the computational results show consistent trends. During the stick-to-slip transition, the rubber deformations grow with the increasing $F_{f x}$. The points on $\partial \mathcal{P}$ start slipping first and then the slipping region propagates to the center portion of $\mathcal{P}$. It is interesting to note that the last portion 


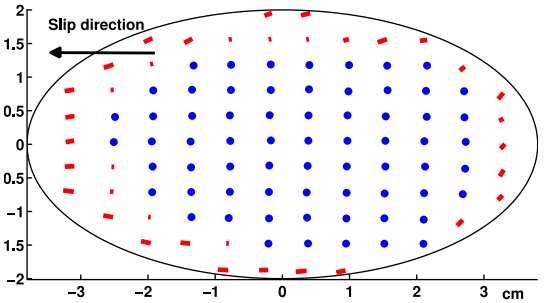

(a)

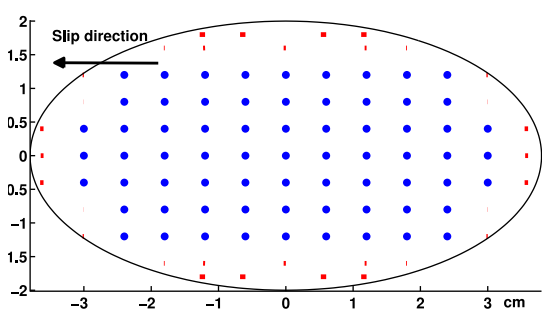

(d)

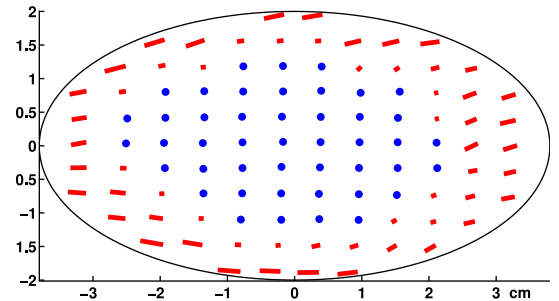

(b)

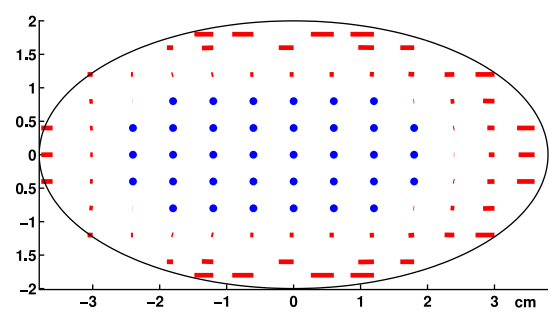

(e)

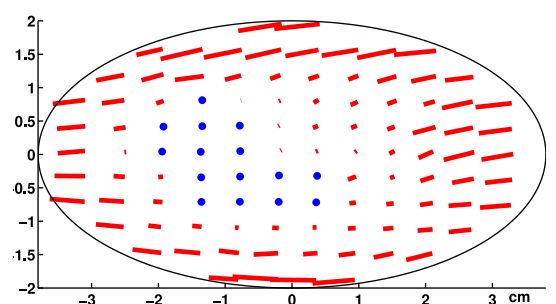

(c)

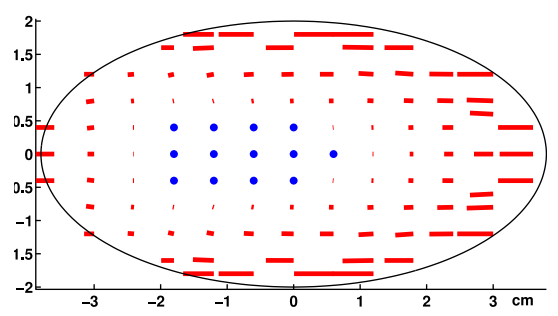

(f)

Fig. 9. Deformation distribution (in $\mathrm{cm}$ ) on $\mathcal{P}$ with various friction forces $F_{f x}$. The blue dots indicate stick point and the red bars indicate slipping displacements. The elliptical contour indicates the contact patch $\mathcal{P}$ and the plotting deformations are magnified five times for clear presentation. Vision-based experiments: (a) $F_{f x}=44.5 \mathrm{~N}$. (b) $F_{f x}=89 \mathrm{~N}$. (c) $F_{f x}=133.5 \mathrm{~N}$. Numerical computation: (d) $F_{f x}=44.5 \mathrm{~N}$. (e) $F_{f x}=89 \mathrm{~N}$. (f) $F_{f x}=133.5 \mathrm{~N}$.

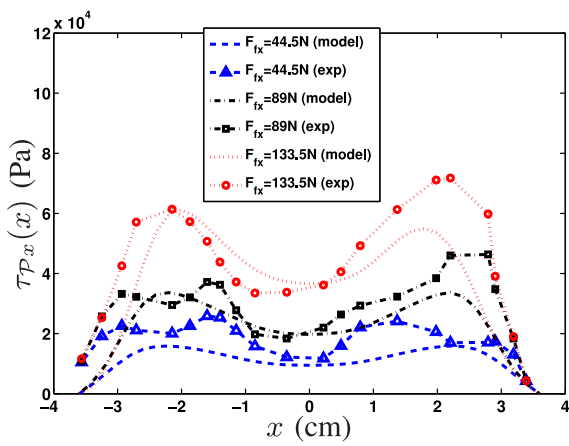

(a)

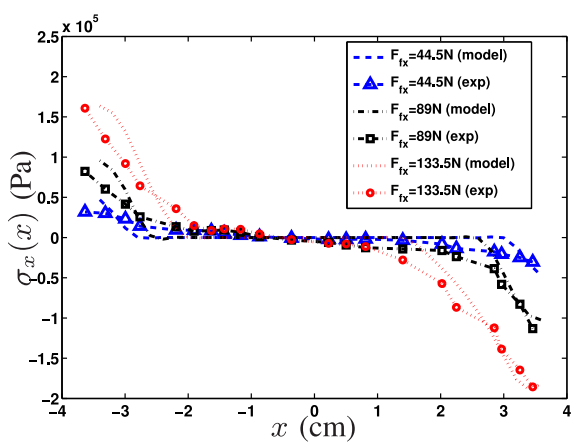

(b)

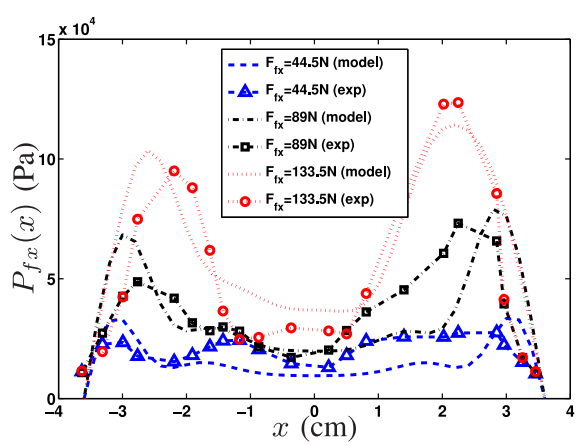

(c)

Fig. 10. Comparison results between model predictions and sensor measurements of stress distributions on $\mathcal{P}$ under various friction forces $F_{f x}$. (a) $\tau_{\mathcal{P} x}(x)$. (b) $\sigma_{x}(x)$. (c) $P_{f x}(x)$.

to slip is not exactly the geometry center of $\mathcal{P}$ but the location close to the trailing edge.

To study the local friction force distributions during the stickslip transition, scanning experiments are conducted by moving the embedded sensor at various locations along the tire center line (i.e., fixed $y=0$ ). At a given location $x$, both the local longitudinal and lateral normalized friction forces $P_{f x}(x)$ and $P_{f y}(x)$ exist. The first set of scanning experiments are conducted under only $F_{f x}$ and in this case, $P_{f y}(x)=0$ along the center line due to the symmetry. Fig. 10(a) and (b) shows the comparison results between the model predictions and the experiments for $\tau_{\mathcal{P} x}(x)$ and $\sigma_{x}(x)$, respectively. The results demonstrate a clear agreement between the model predictions and the experiments. As shown in Fig. 10(a), the distribution of $\tau_{\mathcal{P} x}(x)$ is not uniform on $\mathcal{P}$ and indeed has a similar pattern to the distribution of $P_{n}(x)$. The results in Fig. 10(b) show that $\sigma_{x}(x)$ has larger values close to $\partial \mathcal{P}$ than those around the center of $\mathcal{P}$. The sign of $\sigma_{x}(x)$ has changed: at the leading edge the rubber is under compression (i.e., negative $\sigma_{x}(x)$ ), while at the trailing edge portion, it is under elongation (i.e., positive $\sigma_{x}(x)$ ). This phenomenon also matches the rubber deformation distribution shown in Fig. 9. Fig. 10(c) shows the matching $P_{f x}(x)$ along the center line of $\mathcal{P}$ between the model predictions and the experiments.

Fig. 11(a) shows the consistently matching results between the model predictions and the experiments for $P_{f y}(x)$ along the center line of $\mathcal{P}$ under only friction force $F_{f y}$. Similar to $P_{f x}(x)$ under $F_{f x}$, the results of $P_{f y}(x)$ under $F_{f y}$ show a two-peak distribution pattern. Finally, the experiments are conducted to obtain $P_{f x}(x)$ along the $x$-axis direction under the existence of both $F_{f x}$ and $F_{f y}$. Fig. 11(b) shows the comparison results of $P_{f x}(x)$. In the scanning experiments, constant $F_{f y}=89 \mathrm{~N}$ is applied. These results confirm a similar distribution pattern to that under only applying $F_{f x}$.

\section{E. Discussion}

The distributions $P_{f x}(x)$ and $P_{f y}(x)$ in Figs. 10(c) and 11 demonstrate a similar two-peak trend as $P_{n}(x)$ shown in Fig. 6 for the case of $P_{\text {air }}=69 \mathrm{kPa}$ and $F_{z}=267 \mathrm{~N}$. To determine 


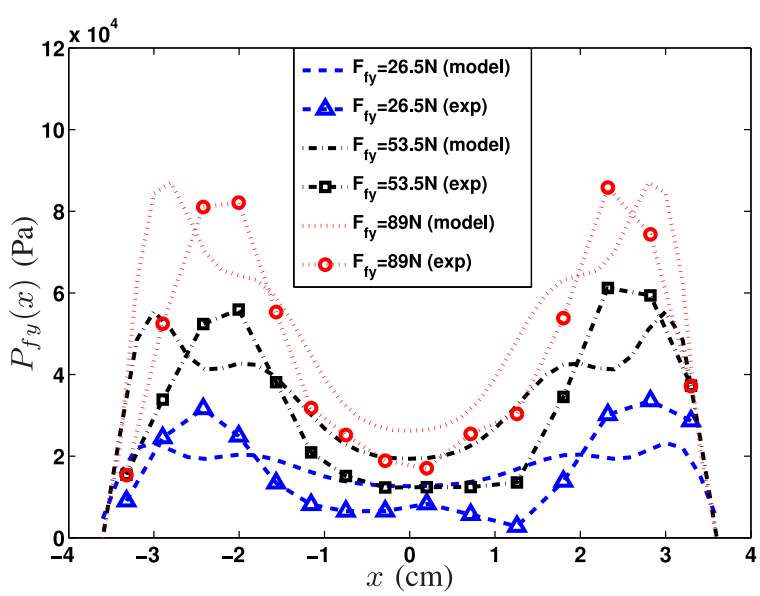

(a)

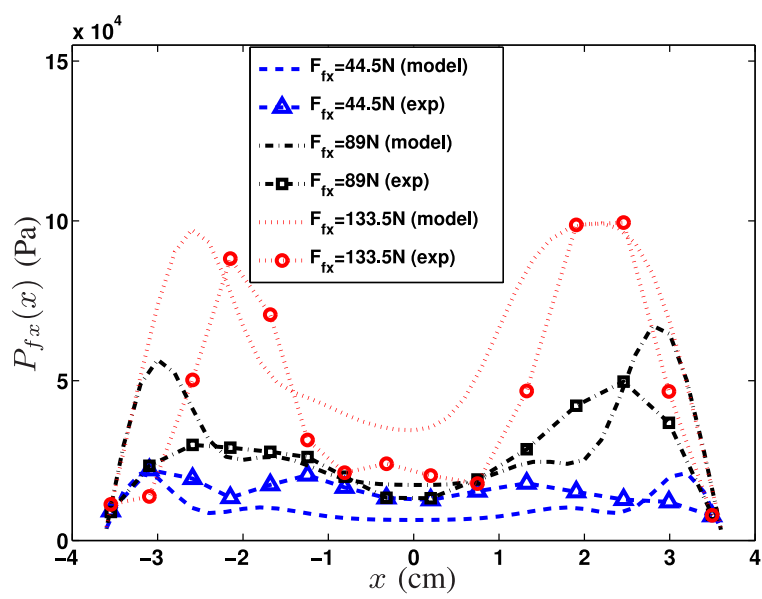

(b)

Fig. 11. (a) Comparison results between model prediction of $P_{f y}(x)$ distribution and sensor measurements under various friction forces $F_{f y}$ along the center line. (b) Comparison of friction force distribution $P_{f x}(x)$ under a combined $F_{f x}$ and $F_{f y}=89 \mathrm{~N}$.

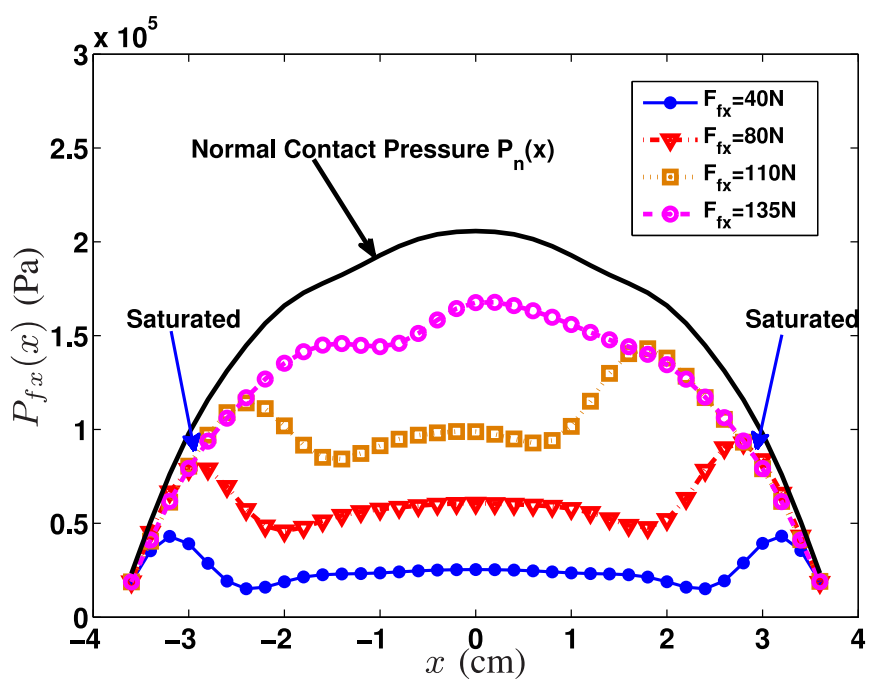

Fig. 12. Friction force distribution $P_{f x}(x)$ along the tire center line under a single-peak normal pressure distribution $P_{n}(x)$.

whether the friction force distribution completely follows the distribution of $P_{n}(x), P_{f x}(x)$ is computed under a single-peak $P_{n}(x)$ distribution by using parameter $\rho=0.86$. Fig. 12 shows the evolution of $P_{f x}(x)$ distributions under increasing $F_{f x}$. It is interesting to see that with increasing $F_{f x}$, the evolution of the distribution of $P_{f x}(x)$ changes its shape: at the beginning of the stick-to-slip transition, the $P_{f x}(x)$ distributions show a twopeak pattern; see the cases of $F_{f x}=40,80$, or $110 \mathrm{~N}$. However, when the full slip starts on $\mathcal{P}$, that is, the case of $F_{f x}=135 \mathrm{~N}$, $P_{f x}(x)$ follows the single-peak shape, similar to that of $P_{n}(x)$.

From the above comparison results, it is concluded that the local friction force distribution is indeed a resultant of both the tire thin-layer structure and the normal pressure distribution. Because of the thin-layer structure, when $F_{f x}$ is small, the boundary portion of $\mathcal{P}$ starts slipping and $P_{f x}(x)$ in this portion reaches the saturation value. That is the reason why a two-peak shape of $P_{f x}(x)$ distribution appears at the beginning of the partial slip stage. With an increasing $F_{f x}$, the entire patch is about to slip, and the $P_{f x}(x)$ distribution follows a similar pattern as the $P_{n}(x)$ distribution due to the kinetic friction relationship, i.e., $P_{f x}(x)=\mu P_{n}(x)$. Therefore, during the stick-to-slip transition, the friction force distribution evolutes from the two-peak shape to the shape of the normal pressure distribution.

Although the small pocket motorcycle tire with the smooth outer surface is used in this study, some contact properties such as the normal contact pressure distribution are similar to those of typical passenger car tires, as shown in [8], [9]. Thus, the use of this tread-free tire can obtain meaningful knowledge of the fundamentals of tire/road interactions.

Compared to the experimental results, the prediction results show some discrepancies for both the normal contact pressure model and the stick-slip friction force model. These differences mainly come from several sources. First, the tire/road contact is more complicated than what the models capture in this paper. The imperfect models lead to some prediction errors. For example, the rubber has the hyperelasticity property and both the proposed normal pressure model and stick-slip model do not incorporate such characteristics. In stick-to-slip experiments, due to the physical constraints, the sensor is not installed at the optimal orientation for superior sensitivity [19]. Finally, the limited accuracy of the low-cost PSECR sensor also leads to some measurement errors.

\section{CONCLUSION AND FUtURE WORK}

In this paper, modeling, analysis, and experiments of tire/road stick-slip interactions were presented. A semi-analytical model was first used to calculate the normal pressure distribution. A beam-spring network model was then proposed to capture the evolution of the complex stick-to-slip transition on the tire contact patch. The proposed modeling and analyses were validated through the developed "smart tire" test platform with the embedded PSECR tire force sensor. The model predictions showed highly agreements with the experiments for both the rubber deformation and the local friction force distributions. The results confirmed that the rubber deformation propagates from 
the boundary to the center of the contact patch during the stickto-slip transition. Moreover, the local friction force distribution on the contact patch was determined by both the normal contact pressure distribution and the thin-layer structure properties.

The dynamic interactions of rotating tires is one of the undergoing research tasks. Integration of the model presented in this paper with other friction models (e.g., LuGre dynamic friction model) is another ongoing development. The outcomes of these development will be reported in the future publications.

\section{ACKNOWLEDGMENT}

The authors would like to thank Prof. L. Liu of Rutgers University for his helpful discussions and Dr. J. M. Block for his help in sharing some computational program samples. Many thanks go to A. Allen for his help in setting up the camera systems in the experiments. The authors are also grateful to the anonymous reviewers and the Technical Editor for their constructive suggestions to improve the quality of the paper.

\section{REFERENCES}

[1] APOLLO Consortium. (2003). "Intelligent tyre systems-State of the art and potential technologies," Technical Research Centre of Finland (VTT), APOLLO Deliverable D7 for Project IST-2001-34372. [Online]. Available: http://www.vtt.fi/apollo

[2] H. B. Pacejka, Tire and Vehicle Dynamics, 2nd ed. Warrendale, PA, USA: SAE, 2006.

[3] G. Gim and P. Nikravesh, "An analytical model of pneumatic tyres for vehicle dynamic simulations. Part I: Pure slips," Int. J. Veh. Design, vol. 11, no. 6, pp. 589-618, 1990.

[4] J. Yi, L. Alvarez, X. Claeys, and R. Horowitz, "Tire/road friction estimation and emergency braking control using a dynamic friction model," Veh. Syst. Dyn., vol. 39, no. 2, pp. 81-97, 2003.

[5] J. Deur, J. Asgari, and D. Hrovat, "A 3D brush-type dynamic tire friction model,” Veh. Syst. Dyn., vol. 42, no. 3, pp. 133-173, 2004.

[6] W. Liang, J. Medanic, and R. Ruhl, "Analytical dynamic tire model," Veh. Syst. Dyn., vol. 46, no. 3, pp. 197-227, 2008.

[7] J. Li, Y. Zhang, and J. Yi, "A hybrid physical/dynamic tire/road friction model,” ASME J. Dyn. Syst., Meas., Control, vol. 135, no. 1, pp. 011007$1-011007-11,2013$.

[8] H. Shiobara, T. Akasaka, and S. Kagami, "Two-dimensional contact pressure distribution of a radial tire in motion," Tire Sci. Technol., vol. 24, no. 4, pp. 294-320, 1996.

[9] S. Kim, P. Nikravesh, and G. Gim, "A two-dimensional tire model on uneven roads for vehicle dynamic simulations," Veh. Syst. Dyn., vol. 46, no. 10, pp. 913-930, 2008.

[10] C. Melchiorri, "Slip detection and control using tactile and force sensors," IEEE/ASME Trans. Mechatronics, vol. 5, no. 3, pp. 235-243, Sep. 2000.

[11] J. Yi, L. Alvarez, and R. Horowitz, "Adaptive emergency brake control with underestimation of friction coefficient," IEEE Trans. Contr. Syst. Technol., vol. 10, no. 3, pp. 381-392, May 2002.

[12] L. Alvarez, J. Yi, L. Olmos, and R. Horowitz, "Adaptive emergency braking control with observer-based dynamic tire/road friction model and underestimation of friction coefficient," ASME J. Dyn. Syst., Meas., Control, vol. 127, no. 1, pp. 22-32, 2005.

[13] J. Ueda, A. Ikeda, and T. Ogagawara, "Grip-force control of an elastic object by vision-based slip-margin feedback during the incipient slip," IEEE Trans. Robot., vol. 21, no. 6, pp. 1139-1147, Dec. 2005.

[14] V.-A. Ho, D. V. Dao, and S. Hirai, "Development and analysis of a sliding tactile soft fingertip embedded with a microforce/moment sensor," IEEE Trans. Robot., vol. 27, no. 3, pp. 411-424, Jun. 2011.

[15] K. L. Johnson, Contact Mechanics. Cambridge, UK: Cambridge Univ. Press, 1987.

[16] J. M. Block and L. M. Keer, "Partial plane contact of an elastic curved beam pressed by a flat surface," ASME J. Tribol., vol. 129, no. 1, pp. 60-64, 2007.

[17] T. Omori1, T. Ishikawa, D. Barthés-Biesel, A.-V. Salsac, J. Walter, Y. Imai, and T. Yamaguchi, "Comparison between spring network models and continuum constitutive laws: Application to the large deformation of a capsule in shear flow," Phys. Rev. E, vol. 83, no. 4, 2011.

[18] B. S. Mahal, D. E. R. Clark, and J. E. L. Simmons, "Mass-spring simulation of deformation in elastic sheet structures," Presence, Teleoper. Virtual Environ., vol. 10, no. 3, pp. 331-342, 2001.

[19] Y. Zhang, J. Yi, and T. Liu, "An embedded local force sensor for insitu tire-road interaction measurements," IEEE Sensors J., vol. 13, no. 5, pp. 1756-1765, 2013.

[20] R. Matsuzaki and A. Todoroki, "Wireless flexible capacitive sensor based on ultra-flexible epoxy resin for strain measurement of automobile tires," Sens. Actuators A, vol. 140, pp. 32-42, 2007.

[21] J. Yi, "A piezo-sensor based "smart tire" system for mobile robots and vehicles," IEEE/ASME Trans. Mechatronics, vol. 13, no. 1, pp. 95-103, Feb. 2008.

[22] L. Beccai, S. Roccella, L. Ascari, P. Valdastri, A. Sieber, M. C. Carrozza, and P. Dario, "Development and experimental analysis of a soft compliant tactile microsensor for anthropomorphic artificial hand," IEEE/ASME Trans. Mechatronics, vol. 13, no. 2, pp. 158-168, Apr. 2008.

[23] Y. Zhang, A. W. Allen, J. Yi, and T. Liu, "Understanding tire/road stick-slip interactions with embedded rubber force sensors," in Proc. IEEE/ASME Int. Conf. Adv. Intell. Mechatronics, Kaohsiung, Taiwan, 2012, pp. 550555.

[24] Y. Zhang and J. Yi, "Tire/road stick-slip interactions: Analysis and experiments," presented at the ASME Dyn. Syst. Control Conf., Ft. Lauderdale, FL, USA, 2012. Paper DSCC2012-8619.

[25] Y. Q. Wang, R. Gnadler, and R. Schieschke, "Two-dimensional contact area of a pneumatic tire subjected to a lateral force," Veh. Syst. Dyn., vol. 23, pp. 149-163, 1994.

[26] S. P. Timoshenko, Theory of Elasticity. New York, NY, USA: McGrawHill, 1970.

[27] T. Maeno, T. Kawamura, and S.-C. Cheng, "Friction estimation by pressing an elastic finger-shaped sensor against a surface," IEEE Trans. Robot. Autom., vol. 20, no. 2, pp. 222-228, Apr. 2004.

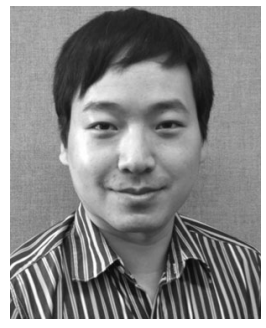

Yizhai Zhang (S'11) received the B.S. and M.S. degrees in information and communication engineering from Xi' an Jiaotong University, Xi' an, China, in 2005 and 2009 , respectively. He is currently working toward the Ph.D. degree in mechanical and aerospace engineering at Rutgers University, Piscataway, NJ, USA.

His current research interests include autonomous robotic systems, dynamic systems and control, intelligent sensing and actuation systems, and mechatronics.

Mr. Zhang is a student member of the American Society of Mechanical Engineers (ASME). He received the Best Student Paper Award at the 2012 IEEE/ASME International Conference on Advanced Intelligent Mechatronics and the 2012 ASME Dynamic Systems and Control Conference.

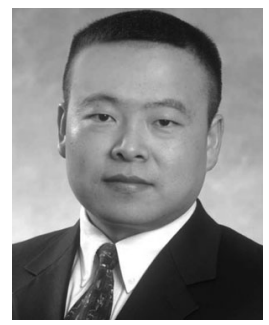

Jingang Yi (S'99-M'02-SM'07) received the B.S. degree in electrical engineering from Zhejiang University, Hangzhou, China, in 1993, the M.Eng. degree in precision instruments from Tsinghua University, Beijing, China, in 1996, and the M.A. degree in mathematics and the Ph.D. degree in mechanical engineering from the University of California, Berkeley, in 2001 and 2002, respectively.

$\mathrm{He}$ is currently an Associate Professor of Mechanical Engineering at Rutgers University, Piscataway, NJ, USA. His research interests include autonomous robotic systems, dynamic systems and control, mechatronics, automation science and engineering, with applications to biomedical systems, civil infrastructure, and transportation systems.

Dr. Yi is a member of the American Society of Mechanical Engineers (ASME). He is a recipient of a 2010 U.S. NSF CAREER Award. He has coauthored papers that have been awarded several best papers at the IEEE/ASME International Conference on Advanced Intelligent Mechatronics, ASME Dynamic Systems and Control Conference, and IEEE International Conference on Robotics and Automation. He currently serves as an Associate Editor for the IEEE TRANSACTIONS ON AUTOMATION SCIENCE AND ENGINEERING, the IFAC journal Control Engineering Practice, and the IEEE Robotics and Automation Society Conference Editorial Board (since 2008). He also served as a Guest Editor for the IEEE TRANSACTIONS ON AUTOMATION SCIENCE AND ENGINEERING in 2009 and an Associate Editor for the ASME Dynamic Systems and Control Division Conference Editorial Board from 2008 to 2010. 